\title{
Communication
}

\section{Multiobjective Optimization of Water Distribution Networks Using Fuzzy Theory and Harmony Search}

\author{
Zong Woo Geem \\ Department of Energy IT, Gachon University, Seongnam 461-701, Korea; \\ E-Mail: zwgeem@gmail.com; Tel.: +82-10-9385-7587. \\ Academic Editor: Enedir Ghisi \\ Received: 30 April 2015 / Accepted: 30 June 2015 / Published: 8 July 2015
}

\begin{abstract}
Thus far, various phenomenon-mimicking algorithms, such as genetic algorithm, simulated annealing, tabu search, shuffled frog-leaping, ant colony optimization, harmony search, cross entropy, scatter search, and honey-bee mating, have been proposed to optimally design the water distribution networks with respect to design cost. However, flow velocity constraint, which is critical for structural robustness against water hammer or flow circulation against substance sedimentation, was seldom considered in the optimization formulation because of computational complexity. Thus, this study proposes a novel fuzzy-based velocity reliability index, which is to be maximized while the design cost is simultaneously minimized. The velocity reliability index is included in the existing cost optimization formulation and this extended multiobjective formulation is applied to two bench-mark problems. Results show that the model successfully found a Pareto set of multiobjective design solutions in terms of cost minimization and reliability maximization.
\end{abstract}

Keywords: multiobjective optimization; water distribution network; fuzzy theory; harmony search; reliability

\section{Introduction}

Up to date, diverse phenomenon-mimicking algorithms (PMAs) have been proposed for the design optimization of water distribution networks (WDNs). These algorithms include genetic algorithm [1], simulated annealing [2], tabu search [3], shuffled frog-leaping algorithm [4], ant colony optimization algorithm [5], harmony search [6], cross entropy [7], scatter search [8], hybrid algorithm [9], 
honey-bee mating optimization [10], differential evolution [11], adaptive cluster covering with local search [12], and Non-Dominated Sorting Genetic Algorithm-II [13].

Although various PMAs have been proposed, these were mostly applied to basic bench-mark WDNs, such as two-loop and Hanoi networks, which are gravity-fed systems with the following optimization formulation:

$$
C=\sum_{i=1}^{P} f\left(D_{i}, L_{i}\right)
$$

where $f(\cdot)$ is cost function which has two arguments of pipe diameter $D_{i}$ and pipe length $L_{i}$; and $P$ is total number of pipe $i$. This cost function is to be minimized while satisfying the following constraints:

$$
\sum Q_{\text {in }}-\sum Q_{\text {out }}=Q_{e}
$$

where $Q_{\text {in }}$ is inflow amount to a specific node; $Q_{\text {out }}$ is outflow amount from the specific node; and $Q_{e}$ is external demand amount at the node. This continuity constraint must be satisfied for every node in a network.

$$
\sum h_{f}=0
$$

where $h_{f}$ is head loss due to pipe friction, which is calculated using Hazen-Williams or Darcy-Weisbach formulae. This energy conservation constraint must be satisfied for every loop in the network.

$$
H_{j} \geq H_{j}^{\min }
$$

where $H_{j}$ is pressure head at node $j ; H_{j}^{\text {min }}$ is minimal pressure head required for node $j$. This minimum pressure constraint must be satisfied for every node in the network.

In addition to the above optimization formulation, one more reliability constraint was recently considered for the water network optimization. Geem et al. [14] added flow velocity constraint for every pipe as follows:

$$
v_{i}^{\min } \leq v_{i} \leq v_{i}^{\max }
$$

where $v_{i}$ is flow velocity through pipe $i$; and $v_{i}^{\min }\left(0.1 \mathrm{~m} / \mathrm{s}\right.$ in this study) and $v_{i}^{\max }(3.0 \mathrm{~m} / \mathrm{s}$ in this study) are respectively lower and upper bounds of flow velocities in pipe $i$. Here, in order to analyze the water network and to obtain hydraulic values such as flowrate, pressure head, and flow velocity, a popular simulator EPANET [15] is used.

Although this flow velocity constraint has been seldom adopted in previous optimization researches [16], it is critical with respect to pipe reliability. Excessive high flow velocity causes pipe erosion and structural vulnerableness when water hammer occurs. Additionally, it causes significant head loss [17] because of the following relationships:

$$
\begin{aligned}
& h_{f} \propto v^{1.85} \text { (Hazen-Williams formula) } \\
& h_{f} \propto v^{m} \text { (Darcy-Weisbach formula) }
\end{aligned}
$$


Here, it should be noted that the friction coefficient in the Darcy-Weisbach formula is a function of the velocity. Thus, the relationship between head loss and velocity depends on the hydraulic regime (the hydraulic exponent $m$ is 2 only in "fully developed turbulent flow"; it ranges from 1.75 to 2 in "transition flow"; it is 1.75 in "hydraulically smooth flow"; and it becomes 1 in "laminar flow").

Meanwhile, minimum velocity condition is required to prevent fine material sedimentation, which sometimes blocks the pipe.

This study intends to move forward one more step in terms of the reliability of flow velocity. Instead of being considered as the constraint, the flow velocity can be more precisely considered using fuzzy theory.

Thus far, researchers have proposed some reliability indexes: Todini [18] enumerated various failures due to mechanical reason (such as pipe break, pump stop, and power outage) and hydraulic reason (such as demand change and pipe aging), and then proposed a resilience index which is a measure to quantify how much a network can overcome the failures; Prasad and Park [19] also adopted the resilience index and simultaneously optimized cost and reliability using genetic algorithm; Prasad and Tanyimboh [20] proposed flow entropy as a surrogate reliability measure, which alleviates the drawbacks of resilience index; Ghajarnia et al. [21] proposed a reliability index based on nodal pressure; and Liu et al. [22] suggested a flow entropy-based reliability index. Atkinson et al. [23] categorized various reliability indicators into four groups such as Resilience Index [18,19], Entropy [24,25], Minimum Surplus Head [26], and Performance [27,28].

However, no reliability index has been proposed yet with respect to flow velocity. Thus, the key point of this work is to present a new reliability index with respect to flow velocity using fuzzy theory.

\section{Fuzzy Theory and Multi-Objective Function}

If we just use the velocity constraint (Equation (5)) in the optimization formulation, there is no difference between the velocity adjacent to minimal allowed velocity $\left(v_{i}^{\min }\right)$ or maximal allowed velocity $\left(v_{i}^{\max }\right)$ and the in-between one. However, if we use fuzzy theory, we can consider how good a velocity is in terms of reliability. For example, the velocity in the center of minimum and maximum bounds should have much higher preference than that located slightly after minimum bound because the velocity near minimum bound may partially have the problems of low velocity. Fuzzy theory can quantify this preference.

Fuzzy theory can be represented as a membership function in Figure 1 if we construct it in symmetric triangular shape. Actually the membership function can have more complicated shape. However, devising more complex shape of membership function requires another extensive task by surveying various hydraulic and practical aspects. Thus, this study adopts the most popular triangular shape to start with. As seen in the figure, velocity reliability (VR) or fuzzy membership degree is zero at $v_{i}=v_{i}^{\min }$ or $v_{i}=v_{i}^{\max }$ while it becomes $100 \%$ at $v_{i}=v_{i}^{\text {mean }}=\left(v_{i}^{\min }+v_{i}^{\max }\right) / 2$. Any in-between velocity has the reliability calculated as follows: 


$$
V R=\left\{\begin{array}{cc}
0 & \text { if } v_{i}<v_{i}^{\text {min }} \\
\frac{v_{i}-v_{i}^{\text {min }}}{v_{i}^{\text {mean }}-v_{i}^{\text {min }}} & \text { if } v_{i}^{\min } \leq v_{i} \leq v_{i}^{\text {mean }} \\
\frac{v_{i}-v_{i}^{\text {max }}}{v_{i}^{\text {mean }}-v_{i}^{\text {max }}} & \text { if } v_{i}^{\text {mean }}<v_{i} \leq v_{i}^{\text {max }} \\
0 & \text { if } v_{i}^{\max }<v_{i}
\end{array} \text { where } v_{i}^{\text {mean }}=\frac{v_{i}^{\text {min }}+v_{i}^{\text {max }}}{2}\right.
$$

Then, total velocity reliability for a network, called velocity reliability index (VRI) in this study, can be calculated by summing all velocity reliabilities in each pipe, as follows:

$$
V R I=\sum_{i=1}^{P} V R_{i}
$$

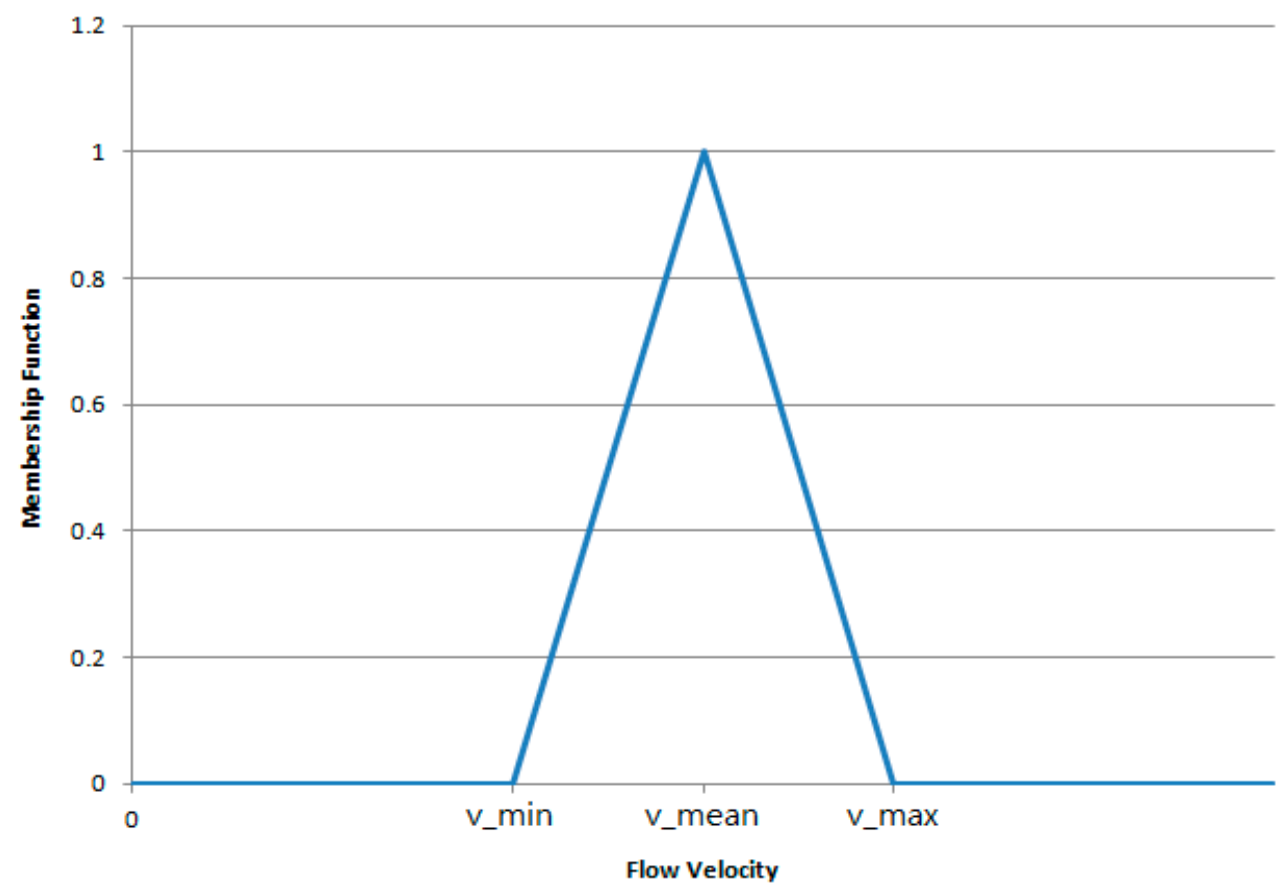

Figure 1. Fuzzy membership function for flow velocity Reliability.

And this optimization problem becomes to have multiobjective functions as follows:

$$
\text { Minimize } C=\sum_{i=1}^{P} f\left(D_{i}, L_{i}\right) \text { and Maximize } V R I=\sum_{i=1}^{P} V R_{i}
$$

Equation (10) substitutes both Equation (1) and Equation (5) for this new bi-objective optimization problem.

\section{Harmony Search Algorithm}

Harmony search algorithm, first proposed by Geem et al. [29], has been continuously developed in terms of both theory and application. HS applications include structural design [30], geotechnical analysis [31], road property analysis [32], groundwater management [33], and project scheduling [34].

HS is similar to other evolutionary or phenomenon-inspired algorithms as a meta-heuristic algorithm. It possesses two basic operators (exploration operator for global search and exploitation 
operator for local search) as other algorithms also have. However, each algorithm has its own uniqueness in term of how it balances the two operators.

Furthermore, HS has its own theoretical background of stochastic derivative [35] to search for a global optimum, which is a new paradigm from the traditional gradient-based derivative in differential calculus. This novel derivative, which is applicable to a discrete variable rather than a continuous one, gives different selection rate to each candidate value for the discrete decision variable, as follows:

$$
\left.\frac{\partial f}{\partial x_{i}}\right|_{x_{i}=x_{i}(k)}=\frac{1}{K_{i}} \cdot P_{\text {Random }}+\frac{n\left(x_{i}(k)\right)}{H M S} \cdot P_{\text {Memory }}+\frac{n\left(x_{i}(k \mp m)\right)}{H M S} \cdot P_{\text {Pitch }}
$$

where $f$ is objective function; $x_{i}$ is discrete decision variable; $x_{i}(k)$ is a specific discrete value for the variable $x_{i}$, which is the element of value set $\left\{x_{i}(1), x_{i}(2), \ldots, x_{i}(k), \ldots, x_{i}\left(K_{i}\right)\right\} ; K_{i}$ is total number of discrete values for the variable $x_{i} ; P_{\text {Random }}$ is probability to consider random selection operation; $P_{\text {Memory }}$ is probability to consider memory consideration operation; $P_{P i t c h}$ is probability to consider pitch adjustment operation; $m$ is neighboring index which has normally one; $n(\cdot)$ is count function which counts the number of the specific discrete value stored in harmony memory (HM); and HMS is size of $\mathbf{H M}$ which is expressed as follows:

$$
\mathbf{H M}=\left[\begin{array}{cccc|c}
x_{1}^{1} & x_{2}^{1} & \cdots & x_{n}^{1} & f\left(\mathbf{x}^{1}\right) \\
x_{1}^{2} & x_{2}^{2} & \cdots & x_{n}^{2} & f\left(\mathbf{x}^{2}\right) \\
\vdots & \cdots & \cdots & \cdots & \vdots \\
x_{1}^{H M S} & x_{2}^{H M S} & \cdots & x_{n}^{H M S} & f\left(\mathbf{x}^{H M S}\right)
\end{array}\right]
$$

Here, the summation of three probabilities $P_{\text {Random }}, P_{\text {Memory }}$, and $P_{\text {Pitch }}$ becomes unity (or 100\%) as follows:

$$
P_{\text {Random }}+P_{\text {Memory }}+P_{\text {Pitch }}=1
$$

Additionally, the cumulative value of the derivatives of all candidate discrete values for each variable $x_{i}$ should be equal to unity:

$$
\left.\sum_{k=1}^{K_{i}} \frac{\partial f}{\partial x_{i}}\right|_{x_{i}=x_{i}(k)}=\frac{\sum_{k=1}^{K_{i}} 1}{K_{i}} P_{\text {Random }}+\frac{\sum_{k=1}^{K_{i}} n\left(x_{i}(k)\right)}{H M S} P_{\text {Memory }}+\left[\frac{\sum_{k=1+m}^{K_{i}+1} n\left(x_{i}(l-m)\right)}{H M S}+\frac{\sum_{k=0}^{K_{i}-m} n\left(x_{i}(l+m)\right)}{H M S}\right] \frac{P_{P i t c h}}{2}=1
$$

In addition to this stochastic derivative, HS has a parameter-setting-free (PSF) process, which gives algorithm users user-friendliness without requiring tedious and time-consuming task of assigning proper values for algorithm parameters [36-38].

The PSF-HS simultaneously manages a new matrix, named OTM (operation type memory), to memorize the type of the operation: 


$$
\left[\begin{array}{cccc}
o t_{1}^{1} & o t_{2}^{1} & \cdots & o t_{n}^{1} \\
o t_{1}^{2} & o t_{2}^{2} & \cdots & o t_{n}^{2} \\
\vdots & \cdots & \cdots & \cdots \\
o t_{1}^{H M S} & o t_{2}^{H M S} & \cdots & o t_{n}^{H M S}
\end{array}\right]
$$

where $o t_{k}^{l}$ denotes operation type, which has one of three values (random selection, memory consideration, or pitch adjustment). Based on OTM, algorithm parameters $P_{\text {Random }}, P_{\text {Memory }}$, and $P_{\text {Pitch }}$ are automatically determined.

HS also has a multiobjective optimization strategy [34]. While the original HS algorithm updates HM based on single objective function as follows:

$$
\mathbf{x}^{\text {Worst }} \notin \mathbf{H M} \wedge \quad \mathbf{x}^{\text {New }} \in \mathbf{H M}
$$

where $\mathbf{x}^{\text {Worst }}$ is worst solution vector stored in $\mathbf{H M}$ and $\mathbf{x}^{\text {New }}$ is newly generated vector using stochastic derivative of Equation (11), the multiobjective HS algorithm updates HM based on two (or more) objective functions as follows:

$$
\mathbf{x}^{\text {Worse }} \notin \mathbf{H M} \wedge \mathbf{x}^{\text {New }} \in \mathbf{H M} \quad \text { if } \mathbf{x}^{\text {New }} \succ \mathbf{x}^{\text {Worse }}
$$

where the symbol $\succ$ represents strict domination. That is, only if the newly generated vector $\mathbf{x}^{\text {New }}$ strictly dominates another vector $\mathbf{x}^{\text {Worse }}$ stored in $\mathbf{H M}$, these two vectors are swapped. Here, strict domination can be expressed as in Equation (18) or (19):

$$
\begin{aligned}
& C\left(\mathbf{x}^{\text {New }}\right)<C\left(\mathbf{x}^{\text {Worse }}\right) \text { And } \operatorname{VRI}\left(\mathbf{x}^{\text {New }}\right) \geq \operatorname{VRI}\left(\mathbf{x}^{\text {Worse }}\right) \\
& C\left(\mathbf{x}^{\text {New }}\right) \leq C\left(\mathbf{x}^{\text {Worse }}\right) \text { And } \operatorname{VRI}\left(\mathbf{x}^{\text {New }}\right)>\operatorname{VRI}\left(\mathbf{x}^{\text {Worse }}\right)
\end{aligned}
$$

Figure 2 shows a flowchart for the aforementioned multiobjective optimization procedure using fuzzy theory and harmony search.

\section{Applications and Results}

The proposed multiobjective HS model was applied to a popular bench-mark problem, two-loop WDN [39]. As shown in Figure 3, it has one reservoir, two loops, six demand nodes, and eight pipes. Because each pipe can choose any diameter from 14 candidates, total solution space becomes $14^{8}=1.48 \times 10^{9}$. With harmony memory size (HMS) $=30$, harmony memory consideration rate $(\mathrm{HMCR})=0.9$, pitch adjustment rate $(\mathrm{PAR})=0.2$, and maximum iterations $=200,000$, HS obtained the results, as shown in Figure 4, after $10 \mathrm{~s}$. The structure of Pareto curve was not changed any more when the maximum iterations were extended up to $1,000,000$.

As seen in Figure 4, the best solution in terms of cost is A $(C=\$ 419,000$, VRI $=5.58)$ and that in terms of reliability is $\mathrm{D}(C=\$ 510,000, \mathrm{VRI}=7.17)$. Figure 3 also provides individual velocity (unit $=\mathrm{m} / \mathrm{s})$ and corresponding VR for the least cost solution $(C=\$ 419,000$, VRI $=5.58)$, which is the identical best solution from the conventional cost-wise single objective optimization.

Figure 5 and Table 1 compares four different Pareto solutions (A to D) chosen from Figure 4. As predicted, the flow velocities of solution $A$ have higher variances from the mean velocity $(1.55 \mathrm{~m} / \mathrm{s})$ than those of solutions B, C, and D in Figure 5. As observed in Table 1, different Pareto solutions have 
different pipe diameters and flow velocities. For example, pipe 8 can have the diameter of one inch (corresponding velocity is $0.32 \mathrm{~m} / \mathrm{s})$ from solution $A$, that of 2 inches $(0.51 \mathrm{~m} / \mathrm{s})$ from solution $\mathrm{B}$, that of 4 inches $(0.94 \mathrm{~m} / \mathrm{s})$ from solution $C$, and that of 8 inches $(1.41 \mathrm{~m} / \mathrm{s})$ from solution $\mathrm{D}$. The less the design cost is, the narrower the diameter is and the slower the velocity is in this case.

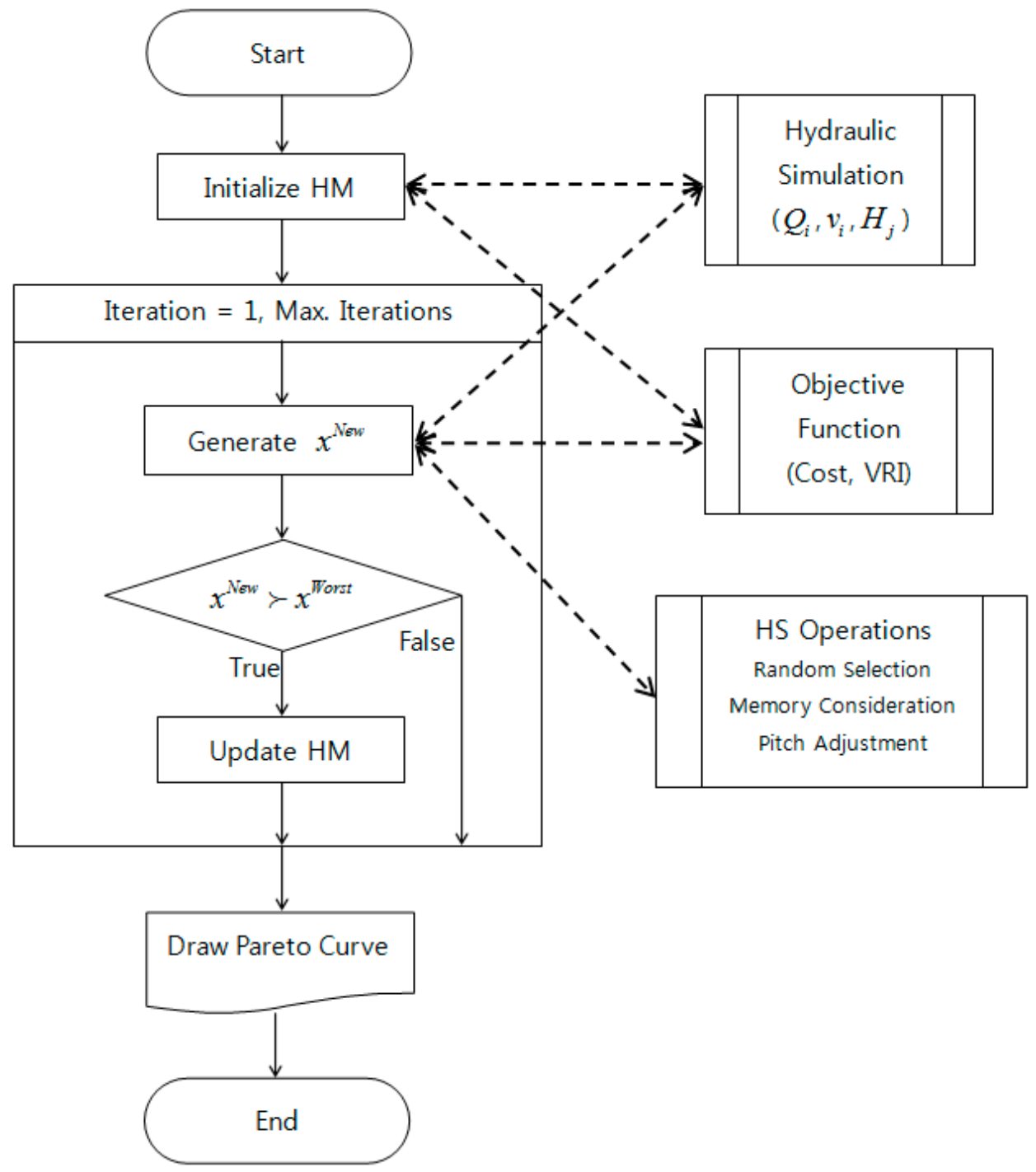

Figure 2. Flowchart of multiobjective optimization.

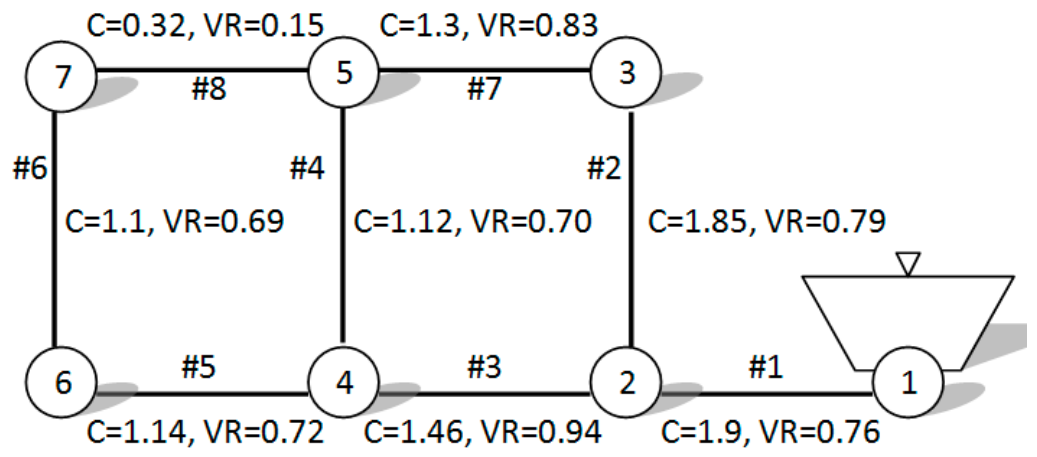

Figure 3. Schematic of two-loop network. 


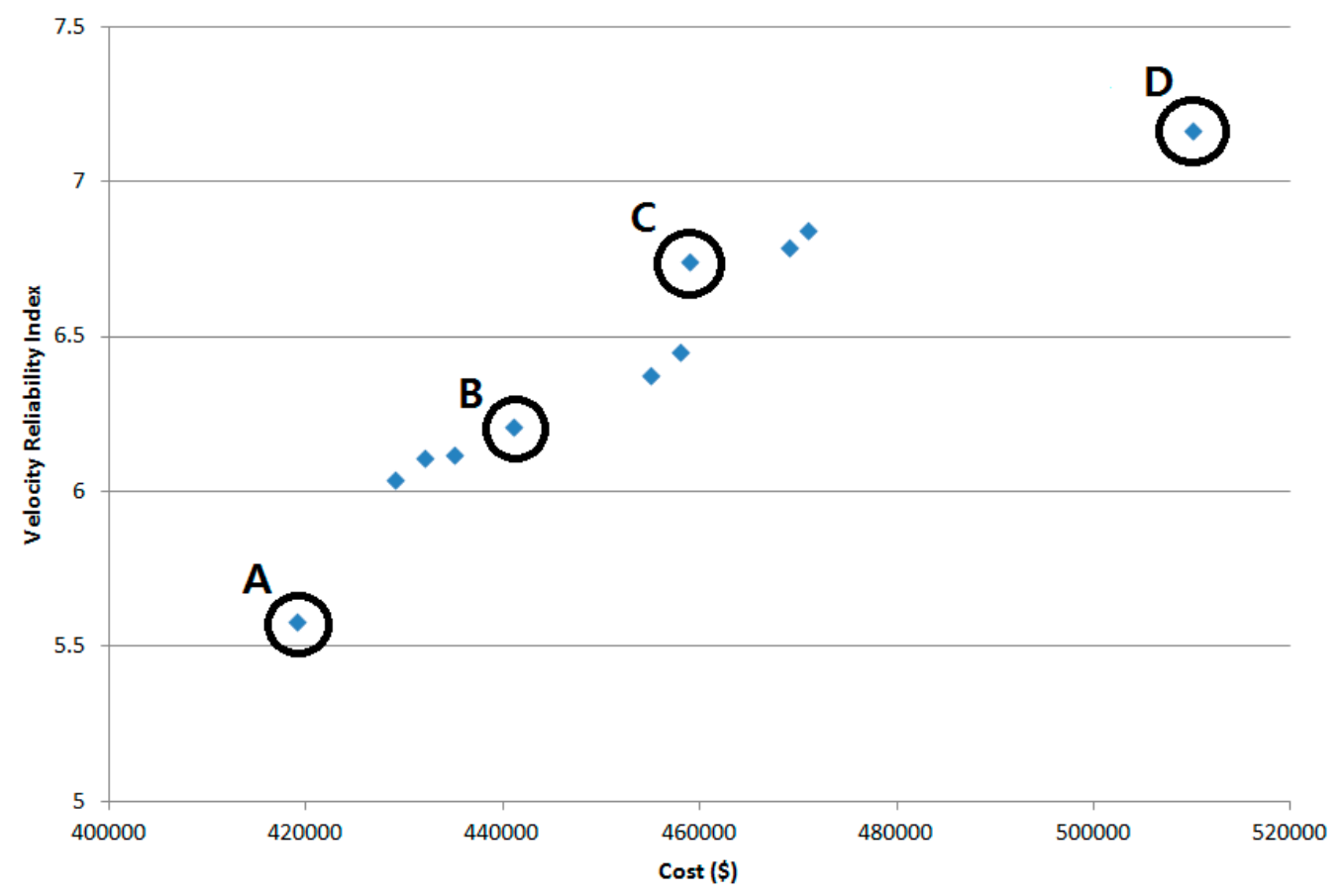

Figure 4. Pareto solutions of two-loop network.

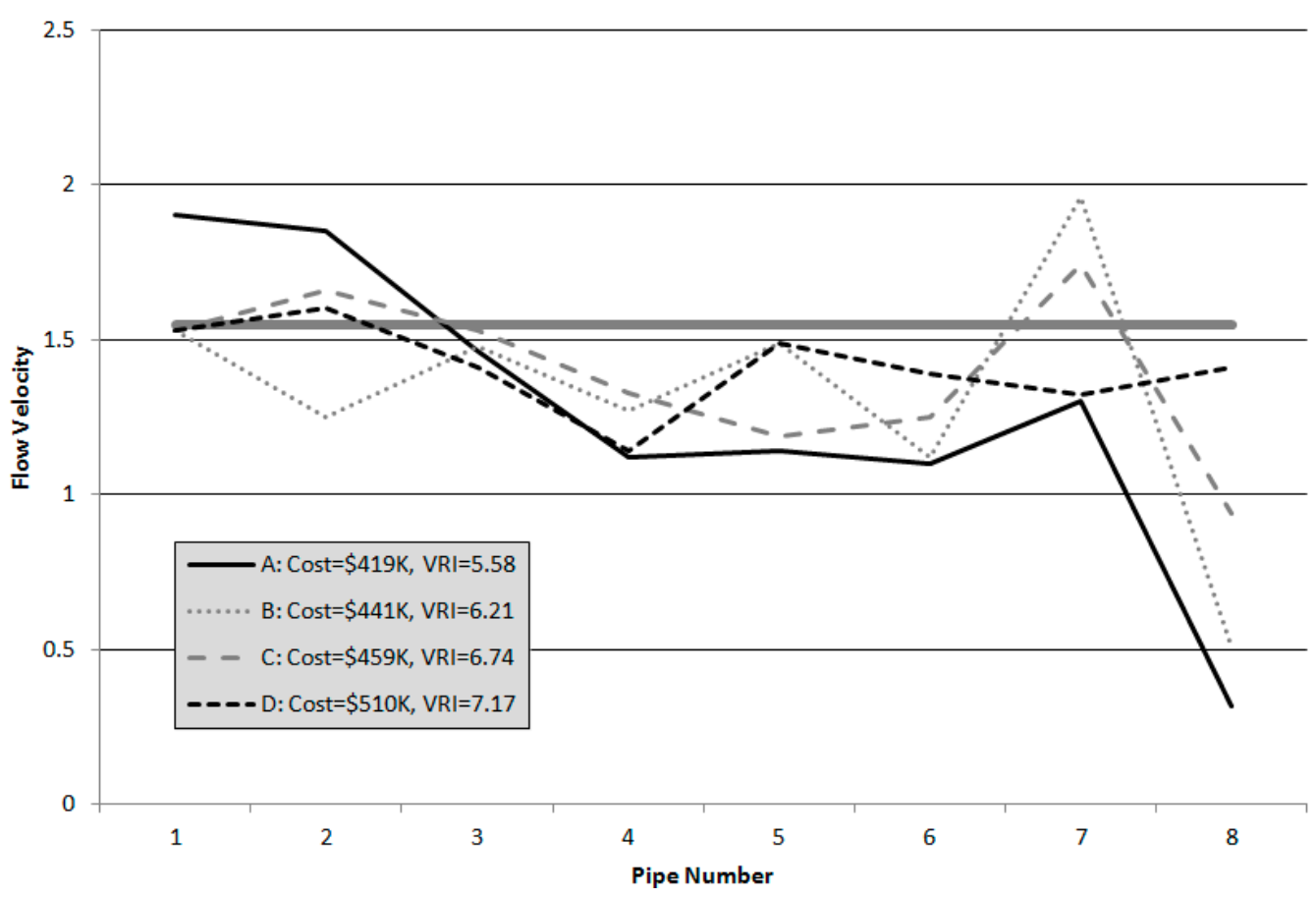

Figure 5. Pareto solutions of two-loop network.

Table 1. Information of four solutions of two-loop network.

\begin{tabular}{|c|c|c|c|c|c|c|c|c|}
\hline \multirow{2}{*}{$\begin{array}{c}\text { Pareto Solution } \\
\text { (Cost, VRI) }\end{array}$} & \multicolumn{8}{|c|}{ Pipe Diameters (inch) and Velocities (m/s) } \\
\hline & 1 & 2 & 3 & 4 & 5 & 6 & 7 & 8 \\
\hline A $(\$ 419 \mathrm{~K}, 5.58)$ & $18(1.9)$ & $10(1.85)$ & $16(1.46)$ & $4(1.12)$ & $16(1.14)$ & $10(1.1)$ & $10(1.3)$ & $1(0.32)$ \\
\hline B $(\$ 441 \mathrm{~K}, 6.21)$ & $20(1.53)$ & $12(1.25)$ & $16(1.48)$ & $4(1.27)$ & $14(1.49)$ & $10(1.12)$ & $8(1.96)$ & $2(0.51)$ \\
\hline $\mathrm{C}(\$ 459 \mathrm{~K}, 6.74)$ & $20(1.53)$ & $10(1.66)$ & $16(1.53)$ & $4(1.33)$ & $16(1.25)$ & $10(1.25)$ & $8(1.74)$ & $4(0.94)$ \\
\hline $\mathrm{D}(\$ 510 \mathrm{~K}, 7.17)$ & $20(1.53)$ & $8(1.6)$ & $18(1.41)$ & $3(1.14)$ & $16(1.49)$ & $12(1.39)$ & $6(1.32)$ & $8(1.41)$ \\
\hline
\end{tabular}


When another loading condition (for example, every nodal demand is reduced by $30 \%$ ) was tested for the least cost solution, VRI was reduced into 4.36 from 5.58 and flow velocity was also reduced in every pipe.

The model was further applied to a real-world problem, Yeosu WDN. As shown in Figure 6, it has one reservoir, 11 loops, 18 demand nodes, and 29 pipes [14]. Because each pipe can choose any diameter from six candidates, total solution space becomes $6^{29}=3.68 \times 10^{22}$.

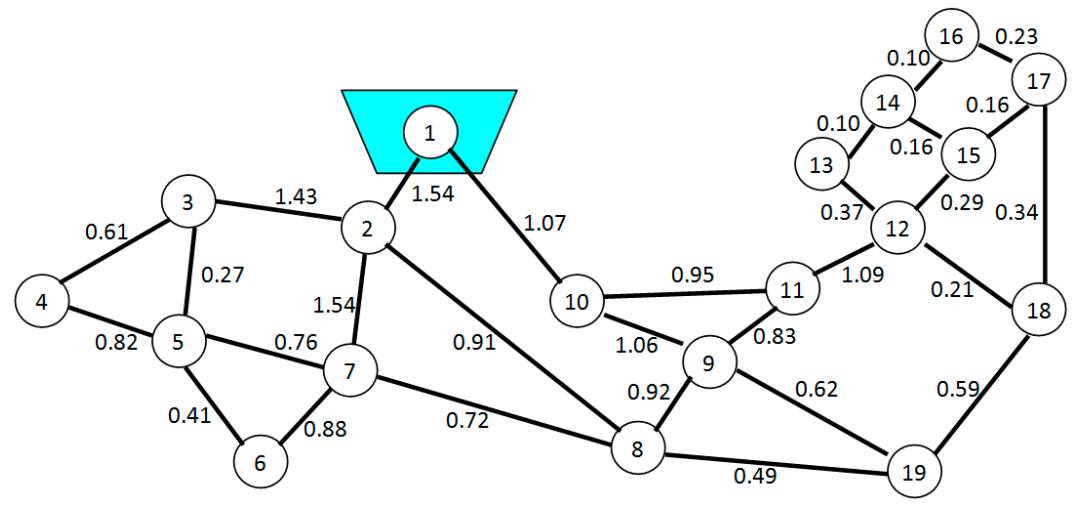

Figure 6. Schematic of Yeosu water distribution network.

With $\mathrm{HMS}=30, \mathrm{HMCR}=0.95, \mathrm{PAR}=0.05$, and maximum iterations $=10^{6}$, HS obtained the results after 2'54". Figure 7 and Table 2 show Pareto solutions of the problem and Figure 6 also provides individual velocity $(\mathrm{m} / \mathrm{s})$ for the least cost solution $(C=211.6$ million Korean Won, VRI $=12.28)$. Decision makers can choose any one design out of five Pareto solutions by considering its budget availability and reliability level. Additionally, if it has special constraint such that it should construct narrow diameter for pipe 12, it may choose the solution 3 (diameter of pipe $12=250 \mathrm{~mm}$ ) rather than other solutions, which have higher diameter $(300 \mathrm{~mm})$ for pipe 12.

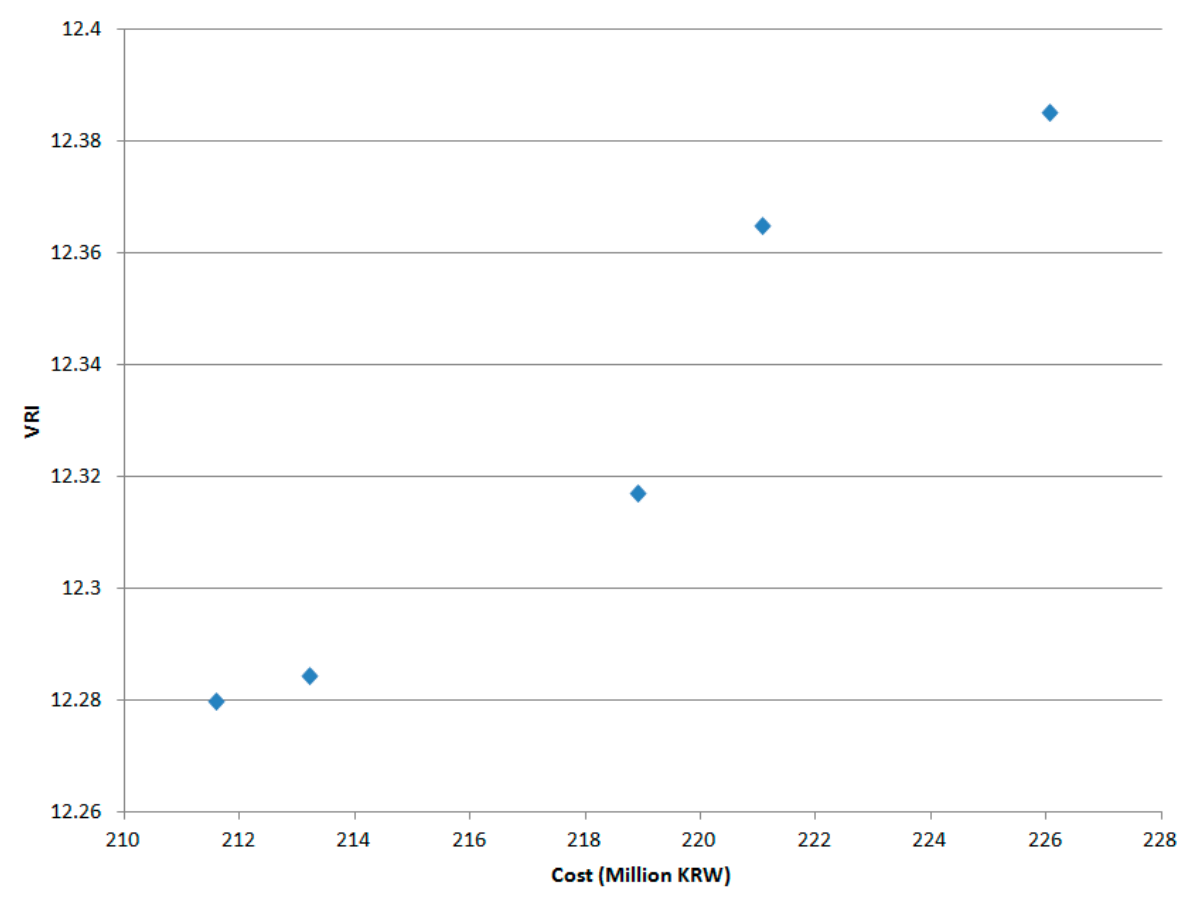

Figure 7. Pareto solutions of Yeosu network. 
Table 2. Information of pareto solutions of Yeosu network.

\begin{tabular}{|c|c|c|c|c|c|}
\hline \multirow{2}{*}{ Pipe Number } & \multicolumn{5}{|c|}{ Pipe Diameters (mm) } \\
\hline & Solution 1 & Solution 2 & Solution 3 & Solution 4 & Solution 5 \\
\hline 1 & 400 & 400 & 400 & 400 & 400 \\
\hline 2 & 200 & 200 & 200 & 200 & 200 \\
\hline 3 & 200 & 200 & 200 & 200 & 200 \\
\hline 4 & 200 & 200 & 200 & 200 & 200 \\
\hline 5 & 200 & 200 & 200 & 200 & 200 \\
\hline 6 & 300 & 300 & 300 & 300 & 300 \\
\hline 7 & 200 & 200 & 200 & 200 & 200 \\
\hline 8 & 200 & 200 & 200 & 200 & 200 \\
\hline 9 & 200 & 200 & 200 & 200 & 200 \\
\hline 10 & 300 & 300 & 300 & 300 & 300 \\
\hline 11 & 200 & 250 & 250 & 200 & 200 \\
\hline 12 & 300 & 300 & 250 & 300 & 300 \\
\hline 13 & 200 & 200 & 200 & 200 & 200 \\
\hline 14 & 200 & 200 & 200 & 200 & 200 \\
\hline 15 & 250 & 200 & 200 & 200 & 200 \\
\hline 16 & 200 & 200 & 200 & 200 & 200 \\
\hline 17 & 200 & 200 & 200 & 200 & 200 \\
\hline 18 & 300 & 300 & 300 & 300 & 300 \\
\hline 19 & 300 & 300 & 350 & 350 & 400 \\
\hline 20 & 200 & 200 & 200 & 200 & 200 \\
\hline 21 & 200 & 200 & 200 & 200 & 200 \\
\hline 22 & 300 & 300 & 250 & 300 & 250 \\
\hline 23 & 200 & 200 & 200 & 200 & 200 \\
\hline 24 & 250 & 250 & 250 & 200 & 250 \\
\hline 25 & 200 & 200 & 200 & 200 & 200 \\
\hline 26 & 200 & 200 & 200 & 200 & 200 \\
\hline 27 & 200 & 200 & 200 & 250 & 200 \\
\hline 28 & 200 & 200 & 200 & 200 & 200 \\
\hline 29 & 200 & 200 & 250 & 250 & 250 \\
\hline Design Cost (Korean Won) & $211.6 \times 10^{6}$ & $213.2 \times 10^{6}$ & $218.9 \times 10^{6}$ & $221.1 \times 10^{6}$ & $226.1 \times 10^{6}$ \\
\hline VRI & 12.280 & 12.284 & 12.317 & 12.365 & 12.385 \\
\hline
\end{tabular}

When this model was applied to another popular benchmark Hanoi network, it could not find proper VRI because velocities of first couple pipes are extremely higher $\left(v_{1}=6.83 \mathrm{~m} / \mathrm{s}\right.$ and $\left.v_{2}=6.53 \mathrm{~m} / \mathrm{s}\right)$ than maximum allowable velocity $(3.0 \mathrm{~m} / \mathrm{s})$ although maximum candidate diameters ( $40 \mathrm{inch})$ were selected for the pipes [14]. Thus, pipe velocities could not be wedged between the lower and upper bounds.

\section{Conclusions}

This study performed WDN design optimization in terms of velocity reliability and cost minimization using fuzzy theory and harmony search. Using fuzzy theory, different degree of reliability was given to flow velocity of each pipe, and this expanded multiobjective formulation was optimized using HS. As a result, a Pareto set of WDN design solutions were successfully obtained. 
The novelty of this study can be the fact that it proposes "preventive" reliability measure while traditional popular resilience indexes are "post-accident" reliability measure. Traditional resilience indexes increase when we add extra energy (for example, more energy to a pump for faster flowrate) to cope with failure situations. However, this surplus energy can be a burden on the system, causing quicker system deterioration. On the contrary, velocity reliability index in this study tries to avoid any excessive velocity, causing fewer burdens on the system.

Based on this pioneering approach in velocity-wise reliability index, more practical researches are expected to enhance system reliability, as well as minimize design cost of real-world WDNs in the future. For example, the less-cost design, with less VRI, may cause more maintenance costs from sediment cleaning or structure break. Thus, if we also consider the maintenance cost, as well as construction cost with respect to life-cycle cost, it will become a more practical design optimization.

Also, other related hybrid techniques such as controlled genetic algorithm [40] and genetic doping algorithm [41] can be considered in the future for devising another velocity-wise reliability index.

\section{Conflicts of Interest}

The author declares that there is no conflict of interests regarding the publication of this paper.

\section{References}

1. Savic, D.A.; Walters, G.A. Genetic algorithms for least-cost design of water distribution networks. J. Water Resour. Plan. Manag. 1997, 123, 67-77.

2. Cunha, M.C.; Sousa, J. Water distribution network design optimization: Simulated annealing approach. J. Water Res. Plan. Manag. 1999, 125, 215-221.

3. Lippai, I.; Heaney, J.P.; Laguna, M. Robust water system design with commercial intelligent search optimizers. J. Comput. Civil. 1999, 13, 135-143.

4. Eusuff, M.; Lansey, K.E. Optimization of water distribution network design using the shuffled frog leaping algorithm. J. Water Res. Plan. Manag. 2003, 129, 210-225.

5. Maier, H.R.; Simpson, A.R.; Zecchin, A.C.; Foong, W.K.; Phang, K.Y.; Seah, H.Y.; Tan, C.L. Ant colony optimization for design of water distribution systems. J. Water Resour. Plan. Manag. 2003, 129, 200-209.

6. Geem, Z.W. Optimal cost design of water distribution networks using harmony search. Eng. Optim. 2006, 38, 259-280.

7. Perelman, L.; Ostfeld, A. An adaptive heuristic cross-entropy algorithm for optimal design of water distribution systems. Eng. Optim. 2007, 39, 413-428.

8. Lin, M.-D.; Liub, Y.-H.; Liua, G.-F.; Chua, C.-W. Scatter search heuristic for least-cost design of water distribution networks. Eng. Optim. 2007, 39, 857-876.

9. Geem, Z.W. Particle-swarm harmony search for water network design. Eng. Optim. 2009, 41, 297-311.

10. Mohan, S.; Jinesh Babu, K.S. Optimal water distribution network design with honey-bee mating optimization. J. Comput. Civil Eng. 2010, 24, 117-126. 
11. Zheng, F.; Simpson, A.R.; Zecchin, A.C. A decomposition and multi-stage optimization approach applied to optimization of water distribution systems with multiple sources. Water Resour. Res. 2013, 49, 380-399.

12. Abebe, A.; Solomatine, D. Application of global optimization to the design of pipe networks. In Proceedings of International Conference on Hydroinformatics, Copenhagen, Denmark, 24-26 August 1998; pp. 989-995.

13. Alfonso, L.; Jonoski, A.; Solomatine, D.P. Multiobjective optimization of operational responses for contaminant flushing in water distribution networks. J. Water Res. Plan. Manag. 2010, 136, $48-58$.

14. Geem, Z.W.; Kim, J.-H.; Jeong, S.-H. Cost efficient and practical design of water supply network using harmony search. Afr. J. Agric. Res. 2011, 6, 3110-3116.

15. Rossman, L.A. EPANET 2: Users Manual; US Environmental Protection Agency: Cincinnati, OH, USA, 2000.

16. Samani, H.M.; Mottaghi, A. Optimization of water distribution networks using integer linear programming. J. Hydraul. Eng. 2006, 132, 501-509.

17. Walski, T.; Chase, D.V.; Savic, D.; Grayman, W.M.; Beckwith, S.; Koelle, E. Advanced Water Distribution Modeling and Management; Haestead Press: Waterbury, CT, USA, 2003.

18. Todini, E. Looped water distribution networks design using a resilience index based heuristic approach. Urban Water 2000, 2, 115-122.

19. Prasad, T.D.; Park, N.-S. Multiobjective genetic algorithms for design of water distribution networks. J. Water Resour. Plan. Manag. 2004, 130, 73-82.

20. Prasad, T.D.; Tanyimboh, T.T. Entropy based design of "Anytown" water distribution network. In Proceedings of Water Distribution Systems Analysis, Kruger National Park, South Africa, 17-20 August 2008; pp. 1-12.

21. Ghajarnia, N.; Haddad, O.B.; Mariño, M.A. Reliability based design of water distribution network (WDN) considering the reliability of nodal pressures. In Proceedings of World Environmental and Water Resources Congress, Kansas City, MS, USA, 17-21 May 2009; pp. 1-9.

22. Liu, H.X.; Savic, D.; Kapelan, Z.; Zhao, M.; Yuan, Y.X.; Zhao, H.B. A diameter-sensitive flow entropy method for reliability consideration in water distribution system design. Water Resour. Res. 2014, 50, 5597-5610.

23. Atkinson, S.; Farmani, R.; Memon, F.A.; Butler, D. Reliability indicators for water distribution system design: Comparison. J. Water Res. Plan. Manag. 2014, 140, 160-168.

24. Awumah, K.; Goulter, I.; Bhatt, S. Assessment of reliability in water distribution networks using entropy based measures. Stoch. Hydrol. Hydraul. 1990, 4, 309-320.

25. Tanyimboh, T.T.; Templeman, A.B. Calculating maximum entropy flows in networks. J. Oper. Res. Soc. 1993, 44, 383-396.

26. Farmani, R.; Walters, G.A.; Savic, D.A. Trade-off between total cost and reliability for anytown water distribution network. J. Water Res. Plan. Manag. 2005, 131, 161-171.

27. Di Nardo, A.; Greco, R.; Santonastaso, G.F.; Di Natale, M. Resilience and entropy indices for water supply network sectorization in district meter areas. In Proceedings of the 9th International Conference on Hydroinformatics, Tianjing, China, 7-11 September 2010. 
28. Raad, D.N.; Sinske, A.N.; Van Vuuren, J.H. Comparison of four reliability surrogate measures for water distribution systems design. Water Resour. Res. 2010, 46, W05524.

29. Geem, Z.W.; Kim, J.H.; Loganathan, G.V. A new heuristic optimization algorithm: Harmony search. Simulation 2001, 76, 60-68.

30. Saka, M.P. Optimum geometry design of geodesic domes using harmony search algorithm. Adv. Struct. Eng. 2007, 10, 595-606.

31. Cheng, Y.M.; Li, L.; Lansivaara, T.; Chi, S.C.; Sun, Y. J. An improved harmony search minimization algorithm using different slip surface generation methods for slope stability analysis. Eng. Optim. 2008, 40, 95-115.

32. Mun, S.; Geem, Z.W. Determination of viscoelastic and damage properties of hot mix asphalt concrete using a harmony search algorithm. Mech. Mater. 2009, 41, 339-353.

33. Ayvaz, M.T. Application of harmony search algorithm to the solution of groundwater management models. Advanc. Water Res. 2009, 32, 916-924.

34. Geem, Z.W. Multiobjective optimization of time-cost trade-off using harmony search. J. Constr. Eng. Manag. 2010, 136, 711-716.

35. Geem, Z.W. Novel derivative of harmony search algorithm for discrete design variables. Appl. Math. Comput. 2008, 199, 223-230.

36. Geem, Z.W.; Sim, K.-B. Parameter-setting-free harmony search algorithm. Appl. Math. Comput. 2010, 217, 3881-3889.

37. Hasancebi, O.; Erdal, F.; Saka, M.P. Adaptive harmony search method for structural optimization. J. Struct. Eng. 2010, 136, 419-431.

38. Geem, Z.W.; Cho, Y.-H. Optimal design of water distribution networks using parameter-setting-free harmony search for two major parameters. J. Water Res. Plan. Manag. 2011, 137, 377-380.

39. Alperovits, E.; Shamir, U. Design of optimal water distribution systems. Water Resour. Res. 1977, 13, 885-900.

40. Lay-Ekuakille, A.; Vendramin, G.; Trotta, A. Spirometric measurement post-processing: Expiration data recovery. Sens. J. IEEE 2010, 10, 25-33.

41. Lay-Ekuakille, A.; Trotta, A. Processing stabilometric data for electronic knee: Training and calibration. In Proceedings of IEEE Instrumentation and Measurement Technology Conference, Austin, TX, USA, 3-6 May 2010; pp. 882-885.

(C) 2015 by the authors; licensee MDPI, Basel, Switzerland. This article is an open access article distributed under the terms and conditions of the Creative Commons Attribution license (http://creativecommons.org/licenses/by/4.0/). 